in vivo $34: 2803-2809$ (2020)

doi:10.21873/invivo.12106

\title{
Predicting Postoperative Events in Patients With Gastric Cancer: A Comparison of Five Nutrition Assessment Tools
}

\author{
SHUN-WEN HSUEH ${ }^{1}$, KENG-HAO LIU ${ }^{2}$, CHIA-YEN HUNG ${ }^{3,4}$, CHUN-YI TSAI $^{2}$, JUN-TE HSU ${ }^{2}$, \\ NGAN-MING TSANG ${ }^{5}$, WILLIAM HARRISON HSUEH ${ }^{6}, \mathrm{CHIEH} \mathrm{YANG}^{7}$ and WEN-CHI $\mathrm{CHOU}^{3}$ \\ ${ }^{1}$ Department of Oncology, Chang Gung Memorial Hospital at Keelung, Keelung, Taiwan, R.O.C.; \\ ${ }^{2}$ Department of Surgery, Chang Gung Memorial Hospital at Linkou and College of Medicine, \\ Chang Gung University, Taoyuan, Taiwan, R.O.C.; \\ ${ }^{3}$ Department of Hematology and Oncology, Chang Gung Memorial Hospital at Linkou and College of Medicine, \\ Chang Gung University, Taoyuan, Taiwan, R.O.C.; \\ ${ }^{4}$ Division of Hema-oncology, Department of Internal Medicine, Mackay Memorial Hospital, Taipei, Taiwan, R.O.C.; \\ ${ }^{5}$ Department of Radiation Oncology, Chang Gung Memorial Hospital at Linkou and College of Medicine, \\ Chang Gung University, Taoyuan, Taiwan, R.O.C.; \\ ${ }^{6}$ School of Medicine, National Defense Medical Center, Taipei, Taiwan, R.O.C.; \\ ${ }^{7}$ Department of Internal Medicine, En-Hua Hospital, New Taipei City, Taiwan, R.O.C.
}

\begin{abstract}
Background/Aim: We compared the adequacy of five nutrition assessment tools with respect to their predictive value in patients with locally advanced gastric cancer (GC) receiving radical surgery. Patients and Methods: Five nutrition assessment tools-Glasgow prognostic score (GPS), malnutritional universal screening tool (MUST), nutritional risk screening, patient generated subjective global assessment (PG-SGA), and prognostic nutritional index (PNI)-were assessed preoperatively for stage III GC patients. The correlation between postoperative events and nutritional status was further analyzed. Results: Most of the nutritional tools accurately predicted length of hospital stay and grade 3 or higher surgical complications, while only the GPS correlated with 30-day readmission and surgical complications. The PG-SGA performed the poorest among the five tools and failed to predict any postoperative event. Conclusion: The application of GPS is recommended as a
\end{abstract}

This article is freely accessible online.

Correspondence to: Wen-Chi Chou, MD, Ph.D., Department of Hematology and Oncology, Chang Gung Memorial Hospital, 5 FuHsing Street, Kwei-Shan Shiang, Taoyuan, Taiwan, R.O.C. Tel: +886 3281200 Ext 2517, Fax: +886 33285818, e-mail: f12986@cgmh.org.tw

Key Words: Nutritional assessment tool, gastric cancer, postoperative complication, Glasgow prognostic score. prognostic index for patients with locally advanced GC prior to radical surgery.

While the treatment of Helicobacter pylori infection has decreased the prevalence of gastric cancer (GC), it remains the fifth most common cancer and was the third leading cause of cancer-related deaths worldwide in $2018(1,2)$. The asymptomatic nature during early stage contributes to its delayed diagnosis and the eventual poor prognosis (3). As radical surgery is the only potential cure for locally advanced GC, nutrition becomes a foremost concern as preoperative malnutrition is well recognized as a risk factor for postoperative morbidity and mortality $(4,5)$.

In cancer patients, malnutrition is often associated with anorexia-cachexia syndrome, characterized by decreased food intake, hypoalbuminemia, weight loss, and muscle tissue wasting (6). While malnutrition is prevalent among patients with advanced malignancy, malnutrition's prognostic role toward postoperative complications or long-term outcome is cancer-type dependent (7). This may be due to the absence of a universal definition of malignancy-related malnutrition and malnutrition's various contributing roles in different cancer types. Malnutrition is prevalent among GC patients due to its advanced stage at diagnosis and the tumor's anatomical location (8). The obstruction of the upper digestive tract directly impedes adequate oral intake which predisposes patients to further nutritional depletion. Despite its curative purpose for GC, gastrectomy is considered a major surgery often that leads to physical stress and wound 
healing, which drives patient's body into a hypermetabolic and catabolic state that may aggravate malnutrition (9). During the treatment of GC, malnutrition is often even more aggravated since vitamin B-12 and iron deficiency are common metabolic sequelae of gastrectomy (10).

In recent clinical practice, nutrition assessment tools that incorporate inflammation factors, such as Glasgow prognostic score and prognostic nutritional index, have also been developed to predict the postoperative morbidity and mortality of various cancers after radical surgeries (11-16). While previous studies have investigated the association between nutrition assessment tools and postoperative complications in GC patients, the results are inconclusive $(10,17)$. The nutrition assessment tools consist of heterogeneous, subjective items, and there is no consensus on the optimal nutrition tool for predicting postoperative complications for locally advanced GC $(17,18)$. Therefore, this retrospective single-center observational study was designed to assess the prognostic value of different nutrition assessment tools based on malnutrition or systemic inflammatory response in patients with locally advanced GC receiving radical surgery.

\section{Patients and Methods}

Patient selection and treatment. In total, 509 consecutive patients with stage III GC who underwent radical gastrectomy and D2 lymph node dissection surgery between 2007 and 2014 at Linkou Chang Gung Memorial Hospital were retrospectively analyzed. Tumor staging was defined according to the 7th edition of the American Joint Committee on Cancer (AJCC) staging system after pathological examination. Patients who underwent palliative surgery, received induction chemoradiotherapy before surgery, or had concurrent active malignancy were excluded. Patients with all the parameters required for Glasgow prognostic score (GPS), malnutritional universal screening tool (MUST), nutritional risk screening (NRS 2002), patient-generated subjective global assessment (PG-SGA), and prognostic nutritional index (PNI) were included. In total, 272 patients were included in the final analysis. For every nutrition assessment tool, patients were stratified into either well-nourished or malnourished status while the cutoff was defined by data obtained from previous studies (19-23). As the patients were stratified into two groups by the five respective nutrition assessment tools, the correlation between postoperative events and nutritional status was further analyzed.

Whether a patient should undergo total or subtotal gastrectomy was decided by the surgeon, based on tumor location and resection margin. This study was approved by the Institutional Review Boards of all the CGMH branches and was performed in compliance with the Helsinki Declaration (1996).

Data collection and follow-up. The details of the study have been described in our previous study (24). In brief, patient and tumor characteristics were retrospectively collected from a prospectively maintained electronic medical record. Subjective variables required for the nutrition assessment tools were obtained from patients or caregivers and clinical laboratory data (complete blood count,
Table I. Score range and data requirement of different nutrition screening tools.

\begin{tabular}{lccccc}
\hline & GPS & MUST & NRS2002 & PG-SGA & PNI \\
\hline Score range & $0-2$ & $0-6$ & $0-7$ & $0-46$ & $*$ \\
Cutoff score & $\geq 1$ & $\geq 2$ & $\geq 3$ & $\geq 9$ & $\leq 45$ \\
for malnutrition & & & & & \\
Objective variable & & & & & \\
$\quad$ Age & & & v & v & \\
CRP & v & & & & \\
Albumin & v & & & & v \\
Lymphocyte & & & & & v \\
BMI & & v & v & & \\
BWL & v & v & v & \\
$\quad$ Disease severity & & v & v & & \\
Subjective variable & & & & \\
$\quad$ Loss of appetite & & v & v & \\
Symptoms & & & v & \\
Physical activity & & & v & \\
\hline
\end{tabular}

* Range of PNI is undefined due to its dependence on albumin and lymphocyte levels. GPS: Glasgow prognostic score; MUST: malnutritional universal screening tool; NRS: nutritional risk screening; PG-SGA: patient generated subjective global assessment; PNI: prognostic nutritional index; CRP: c-reactive protein; BMI: body mass index; BWL: body weight loss.

biochemistry including serum albumin and C-reactive protein) were acquired prior to surgery. All postoperative events, including (length of hospital stay) LOS, surgical complication, postoperative 30-day readmission rate, and surgical mortality were recorded by retrospective chart review. Surgical complications were defined using the Clavien-Dindo Classification, which classified complications of grade 3 or higher as severe complications (25). The overall survival time was defined as the time from the date of surgery to the date of death by any cause or the last objective information registered in the medical chart. All patients were followed up until death or until 6/30/2016.

Glasgow prognostic score. A GPS score, calculated from the Creactive protein (CRP) and albumin levels obtained prior to surgery, was assigned to each patient. Patients with both an elevated CRP $(>10.0 \mathrm{mg} / \mathrm{dl})$ and hypoalbuminemia $(<3.5 \mathrm{~g} / \mathrm{dl})$ were assigned a score of 2 (19). Patients with only one of the two abnormal value were assigned a score of 1 . Patients who had both CRP and albumin levels within normal ranges were assigned a score of 0 . Patients with a GPS score $\geq 1$ were assigned to the malnourished group.

Malnutritional universal screening tool. The MUST score is calculated by patient's BMI, unplanned weight loss, and acute disease effect (20). While a score is assigned for each category, MUST score is the sum of the scores where a value $\geq 2$ is deemed as high risk of malnutrition and patients with such scores were assigned to the malnourished group in our study.

Nutritional risk screening. In nutritional risk screening, patients were scored for each of the two components, 1) nutritional status (BMI and weight loss) and 2) disease severity, according to whether they are absent, mild, moderate, or severe, resulting in a combined 
Table II. Clinicopathological characteristics of the 272 patients with stage III gastric cancer.

\begin{tabular}{lc}
\hline Characteristic & $\mathrm{N}(\%)$ \\
\hline Median age, year (range) & $65.7(26.3-97.2)$ \\
Male sex & $176(64.7)$ \\
ECOG performance scale & \\
0 or 1 & $216(79.4)$ \\
2 & $44(16.2)$ \\
3 & $12(4.4)$ \\
Charlson comorbidity index & \\
0 & $120(44.1)$ \\
1 & $97(35.7)$ \\
2 & $35(12.9)$ \\
$>2$ & $20(7.4)$ \\
Median BMI, kg/m² (range) & $22.2(14.2-33)$ \\
Body weight loss & $176(64.7)$ \\
$<5 \%$ & $96(35.3)$ \\
$\geq 5 \%$ & \\
CEA, ng/dl & $231(84.9)$ \\
$<5$ & $41(15.1)$ \\
$\geq 5$ & \\
CA19-9, ng/dl & $223(82.0)$ \\
$\leq 37$ & $49(18.0)$ \\
$>37$ & \\
T-classification & $9(3.3)$ \\
2 & $101(37.1)$ \\
3 & $162(59.6)$ \\
4 & \\
N-Classification & $44(1.5)$ \\
0 & $18(6.6)$ \\
1 & $77(28.3)$ \\
2 & $103(37.9)$ \\
$3 \mathrm{a}$ & $70(25.7)$ \\
\hline $\mathrm{b}$ & \\
\hline & \\
&
\end{tabular}

ECOG: Eastern Cooperative Oncology Group; BMI: body mass index; CEA: carcinoembryonic antigen; CA19-9: carbohydrate antigen 19-9; AJCC: American Joint Committee on Cancer.

score of 0-6 (21). Patients with a total score of $\geq 3$ were classified as nutritionally at-risk in the original design and assigned to the malnourished group in our study.

Patient generated subjective global assessment. The PG-SGA score is composed of patient-generated historical components (weight history, food intake, symptoms, activities and function) and professional assessment (diagnosis, age, metabolic stress, and physical exam) (22). A numeric score $\geq 9$ required nutrient intervention in the original design, and these patients were assigned to the malnourished group in our study.

Prognostic nutritional index. The PNI was calculated using the following formula: $10 \times$ serum albumin value $(\mathrm{g} / \mathrm{dl})+0.005 \times$ total lymphocyte count in the peripheral blood (per $\mathrm{mm}^{3}$ ) (23). Patients with PNI lower than 45 were considered malnourished in our study.

Summary of nutrition assessment tools. Data requirements for each nutrition assessment tool are presented in Table I. Of the five nutrition assessment tools, GPS, MUST, and PNI required only
Table III. Statistical significance of correlation between patient characteristics and nutritional parameters.

\begin{tabular}{|c|c|c|c|c|c|}
\hline Item & GPS & MUST & NRS 2002 & PG-SGA & PNI \\
\hline $\begin{array}{l}\mathrm{N} \text { of malnutrition } \\
\text { patients }(\%)\end{array}$ & $\begin{array}{c}172 \\
(63.2 \%)\end{array}$ & $\begin{array}{c}118 \\
(43.4 \%)\end{array}$ & $\begin{array}{c}56 \\
(20.6 \%)\end{array}$ & $\begin{array}{c}72 \\
(26.8 \%)\end{array}$ & $\begin{array}{c}58 \\
(21.3 \%)\end{array}$ \\
\hline $\begin{array}{l}\text { Age } \\
(\geq 65 \text { years old })\end{array}$ & 0.07 & 0.37 & * & 0.12 & 0.013 \\
\hline BMI $\left(\leq 18.5 \mathrm{~kg} / \mathrm{m}^{2}\right)$ & 0.18 & $<0.001$ & 0.17 & 0.23 & 0.91 \\
\hline $\mathrm{BWL}(\geq 5 \%)$ & $<0.001$ & $*$ & $*$ & * & 0.64 \\
\hline $\mathrm{CRP}(\geq 10 \mathrm{mg} / \mathrm{l})$ & $*$ & 0.017 & 0.014 & 0.79 & 0.038 \\
\hline $\begin{array}{l}\text { Albumin } \\
(\leq 3.5 \mathrm{gm} / \mathrm{dl})\end{array}$ & $*$ & 0.021 & $<0.001$ & $<0.001$ & * \\
\hline $\begin{array}{l}\text { Lymphocyte } \\
(\geq 10000 / \mu \mathrm{l})\end{array}$ & 0.09 & 0.31 & 0.65 & 0.96 & $*$ \\
\hline $\mathrm{CEA}(\geq 5 \mathrm{ng} / \mathrm{ml})$ & 0.98 & 0.54 & 0.55 & 0.44 & 0.19 \\
\hline CA19-9 (>37 U/ml) & 0.32 & 0.58 & 0.13 & 0.08 & 0.004 \\
\hline $\operatorname{ECOG}(0,1 v s .2,3)$ & 0.026 & 0.18 & $<0.001$ & 0.18 & 0.30 \\
\hline $\mathrm{CCI}$ & 0.26 & 0.16 & $<0.001$ & 0.31 & $<0.001$ \\
\hline T-classification & 0.002 & 0.08 & 0.75 & 0.86 & 0.17 \\
\hline $\mathrm{N}$-Classification & 0.81 & 0.60 & 0.55 & 0.08 & 0.90 \\
\hline AJCC tumor stage & 0.002 & 0.008 & 0.26 & 0.11 & 0.21 \\
\hline Operation method & 0.44 & 0.024 & 0.20 & 0.66 & 0.18 \\
\hline Resection margin & 0.011 & $<0.001$ & 0.002 & 0.13 & 0.70 \\
\hline
\end{tabular}

*Denotes variable originally designated from the respective nutrition assessment tool; thus, the significance of the correlation was not calculated. BMI: Body mass index; BWL: body weight loss; CRP: Creactive protein; CEA: carcinoembryonic antigen; CA19-9: carbohydrate antigen 19-9; ECOG: Eastern Cooperative Oncology Group; CCI: Charlson comorbidity index; AJCC: American Joint Committee on Cancer; GPS: Glasgow prognostic score; MUST: malnutritional universal screening tool; NRS 2002: nutritional risk screening; PG-SGA: patient generated subjective global assessment; PNI: prognostic nutritional index.

clinical data or assessment, while NRS2002 and PG-SGA also relied on information from patients or caregivers.

Statistical analysis. Demographic data were analyzed as median and 95\% confidence intervals (CI). The differences between the nutritional status/clinical variables and postoperative adverse event was determined using the Pearson chi-squared $\left(\chi^{2}\right)$ test or Fisher's exact test. SPSS 17.0 software (SPSS Inc., Chicago, IL, USA) was used for statistical analysis. All statistical assessments were two sided, and a $p$-value of $<0.05$ was considered statistically significant.

\section{Results}

Patient's basic characteristics are presented in Table II. Of the 272 included patients, 176 were men and 96 were women. The median age was 65.7 years (range $=26.3-97.2$ ) and $79.2 \%$ of the patients had an Eastern Cooperative Oncology Group (ECOG) performance of 0 or 1. The prevalence of malnutrition according to each nutrition assessment tool was $63.2 \%, 43.4 \%, 20.6 \%, 26.8 \%$, and $21.3 \%$ according to GPS, MUST, NRS 2002, PG-SGA, and 
Table IV. Statistical significance of correlation between nutritional parameters and postoperative events $(n=461)$.

\begin{tabular}{|c|c|c|c|c|c|}
\hline $\mathrm{N}$ of events $(\%)$ & $\begin{array}{c}\text { LOS } \\
20(18-22)^{*}\end{array}$ & $\begin{array}{c}\text { 30-day readmission } \\
34(12.5 \%)\end{array}$ & $\begin{array}{c}\text { Surgical mortality } \\
7(2.6 \%)\end{array}$ & $\begin{array}{c}\text { Any surgical complication } \\
111(40.8 \%)\end{array}$ & $\begin{array}{c}\text { Surgical complication } \geq \text { grade } 3 \\
48(17.6 \%)\end{array}$ \\
\hline \multicolumn{6}{|l|}{ Single item } \\
\hline Age $\geq 65$ years & 0.004 & 0.039 & 0.28 & $<0.001$ & 0.007 \\
\hline $\mathrm{CRP} \geq 10 \mathrm{mg} / 1$ & 0.001 & 0.003 & 0.14 & 0.001 & 0.005 \\
\hline Albumin $\leq 3.5 \mathrm{gm} / \mathrm{dl}$ & 0.32 & 0.48 & 0.010 & 0.38 & 0.25 \\
\hline Lymphocyte $\geq 10000 / \mu l$ & 0.25 & 0.21 & 0.31 & 0.28 & 0.15 \\
\hline $\mathrm{BMI} \leq 18.5 \mathrm{~kg} / \mathrm{m}^{2}$ & 0.40 & 0.07 & 0.31 & 0.68 & 0.99 \\
\hline $\mathrm{BWL} \geq 5 \%$ & $<0.001$ & 0.99 & 0.22 & 0.47 & 0.09 \\
\hline \multicolumn{6}{|l|}{ Nutritional tool } \\
\hline GPS $\geq 1$ & 0.001 & 0.013 & 0.21 & 0.006 & 0.001 \\
\hline MUST $\geq 2$ & 0.001 & 0.52 & 0.46 & 0.23 & 0.047 \\
\hline NRS $2002 \geq 3$ & 0.001 & 0.36 & 0.14 & 0.12 & 0.044 \\
\hline PG-SGA $\geq 9$ & 0.38 & 0.64 & 0.92 & 0.44 & 0.75 \\
\hline $\mathrm{PNI}>45$ & 0.033 & 0.14 & 0.64 & 0.92 & 0.39 \\
\hline
\end{tabular}

*Indicates median (95\% confidence interval). Statistically significant differences are shown in bold. LOS: Length of hospital stay; CRP: C-reactive protein; BMI: body mass index; BWL: body weight loss; GPS: Glasgow prognostic score; MUST, malnutritional universal screening tool; NRS 2002: nutritional risk screening; PG-SGA: patient generated subjective global assessment; PNI: prognostic nutritional index.

PNI, respectively. The variables needed for the nutrition assessment tools were also examined. In the whole cohort, C-reactive protein (CRP) levels were $>10 \mathrm{ng} / \mathrm{dl}$ in $58.8 \%$ of patients and hypoalbuminemia $(<3.5 \mathrm{~g} / \mathrm{dl})$ was noted in $19.1 \%$ of patients. The median body mass index was 22.2 $\mathrm{kg} / \mathrm{m}^{2}$ and more than a third of the patients experienced body weight loss $\geq 5 \%$.

Table III presents the statistical significance of correlation between patient characteristics and nutrition assessment tools. Apart from the variables originally designated from the respective nutrition assessment tools, several parameters showed significant correlation between patient characteristics and the nutrition assessment tool. GPS showed statistical correlation with body weight loss $(p<0.001)$, ECOG performance $(p=0.026)$, T-classification $(p=0.002)$, AJCC tumor stage $(p=0.002)$, and resection margin $(p=0.011)$. MUST showed correlation with CRP $(p=0.017)$, albumin $(p=0.021)$, BMI $(p<0.001)$, AJCC tumor stage $(p=0.008)$, operation method $(p=0.024)$, and resection margin $(p<0.001)$. NRS 2002 showed correlation with CRP $(p=0.014)$, albumin $(p<0.001)$, ECOG $(p<0.001)$, CCI $(p<0.001)$, and resection margin $(p=0.002)$. PG-SGA showed correlation with albumin $(p<0.001)$. PNI was correlated with age $(p=0.013)$, CRP $(p=0.038)$, CCI $(p<0.001)$, and CA19-9 $(p=0.004)$.

Significance between postgastrectomy events and nutritional parameters is presented in Table IV. While age and CRP levels were correlated with 30-day readmission ( $p=0.039, p=0.003$, respectively), surgical complications $(p<0.001, p=0.001$, respectively), and complication grading $>3$ ( $p=0.007, p=0.005$, respectively), albumin levels were correlated with surgical mortality $(p=0.010)$. Furthermore, CRP and BWL were both correlated with LOS $(p=0.001$, $p<0.001$, respectively). Meanwhile, leukocytosis did not correlate with any postoperative events. Of the nutrition assessment tools, GPS was correlated with 30-day readmission ( $p=0.013)$, surgical complication $(p=0.006)$, and complication grading $(p=0.001)$. MUST and NRS 2002 showed correlation with complication grading of $>3$ ( $p=0.047, p=0.044)$. All the nutrition assessment tools except PG-SGA correlated with LOS. PG-SGA showed no correlation with any of the short-term postoperative events.

\section{Discussion}

While malnutrition and malignancy are frequently related, the prognostic role of malnutrition in many specific cancers is often undefined. A convenient nutrition assessment tool with good prognostic value may be beneficial for medical decision making. Few studies have made direct comparisons among different nutrition assessment tools regarding their prognostic value in predicting the postoperative complications in GC patients after radical gastrectomy (17, 18 , 26).To identify the best correlating parameter to malnutrition in locally advanced GC, this study crossanalyzed five common nutrition assessment tools-GPS, MUST, NRS 2002, PG-SGA, and PNI-with the parameters used within each nutrition assessment tool. Comprehensive nutritional data were collected retrospectively from 272 patients with stage III GC treated at a high-volume medical center in Taiwan. This study revealed the significant prevalence of malnutrition among patients initially diagnosed with locally advanced GC, ranging from $20.6 \%$ to $63.2 \%$, as evaluated by the different nutritional assessment tools. Furthermore, GPS was the most sensitive and useful tool for 
predicting short-term events in terms of LOS, 30-day readmission rate, surgical complication, and severe surgical complications of gastrectomy. Both MUST and NRS showed prognostic value in predicting the LOS and severe surgical complications of gastrectomy. PNI showed correlation with LOS while PG-SGA failed to determine any short-term complications. While PG-SGA has been a valued prognostic tool in general oncology practice, it predominantly relies on subjective answers from patients compared to other nutritional tools. PG-SGA's inability to demonstrate significance in this study may due to the introverted nature of Taiwanese patients that may have affected the end score of the PG-SGA.

Different nutrition assessment tools require different sources of data input. Three of five tools, including GPS, MUST, and PNI, solely used objective variables for nutrition assessment, while the other two (NRS 2002 and PG-SGA) involved both objective and subjective variables for assessment. Subjective variables often entail patient participation, which may be time consuming and require extra manual work force. They may also yield high variance due to patients' different perceptions of sensation and unaccounted emotional weight. Thus, this might explain the unsatisfactory predictive value of the NRS 2002 and PGSGA in our study.

To further distinguish the nutrition assessment tools, this study cross-analyzed the individual parameters used within the five nutrition assessment tools and the nutrition assessment tools themselves. Among the evaluated parameters, albumin, BWL, and CRP were prominent factors that correlated with the nutrition assessment tools. Apart from the GPS and PNI, which originally incorporated albumin for evaluation, albumin revealed independent association with MUST, PG-SGA, and NRS. CRP, which reflects systemic inflammation, also revealed independent relevance with MUST, NRS, and PNI. As BWL is incorporated in MUST, NRS, and PG-SGA, reflecting its importance in the evaluation of malnutrition, BWL was also independently associated with elevated GPS. Even though BMI showed relevance with MUST score, MUST incorporated BWL, which may confound the correlation since BWL and BMI both rely heavily on weight measurements. While these nutrition assessment tools have shown prognostic value in various cancers, albumin, BWL, and CRP stand out among the cross-analyzed parameters in the case of locally advanced GC. This may be explained by albumin, BWL, and CRP's significant reflection of malnutrition and systemic inflammation according to previous studies $(7,15,20)$.

The associations between the short-term postoperative events and the individual parameters used in each nutrition assessment tool were also analyzed. While albumin and CRP were independently associated with different aspects of short-term postoperative events, GPS, which incorporated both albumin and CRP, stood out among the nutrition assessment tools and showed the best correlation with shortterm postoperative events. The NRS and MUST both showed prominence in predicting surgical complication grade of $>3$. However, GPS stood out in 4 of the 5 events and had $p$-value of 0.001 in predicting surgical complication grade of $>3$. Interestingly, all the nutrition assessment tools except PGSGA correlated with LOS. Even though one out of the five postoperative outcomes showed correlation with almost all the nutrition assessment tools, this nonetheless demonstrates the ability of nutrition assessment tools to recognize malnourished patients who may need extra medical attention during their hospital stay.

While both the GPS and PNI consider only objective variables, they also include albumin as one of the parameters. Interestingly, the PNI did not demonstrate predictive value for any postoperative events in this study. However, a study by Lee et al. demonstrated the significance of PNI in predicting short- and long-term postoperative outcomes in a large number of studies (23). Our study and Lee's study both signify albumin's prognostic value. As both CRP and lymphocytes account for inflammatory response, we believe that inflammatory response contributed to its prognostic value along with malnutrition. The discrepancy between the two studies may due to the smaller study sample in our study. While the prognostic value of GPS is pronounced in this study, a study with a larger cohort to verify the prognostic value of GPS is encouraged.

Our previous study has indicated that patients with GPS 2 had an appalling in-hospital mortality rate of $10 \%$, while for patients with GPS 0 and GPS 1, the in-hospital mortality rates were $1.0 \%$ and $1.5 \%$ respectively $(p=0.006)$, indicating ostensibly poor prognosis surgery in patients with GPS 2 (24). However, no in-hospital mortality was observed in association with the GPS in this study. The reason for the discrepancy between the two studies is the simplification of the nutrition assessment tools that was necessary for this study. As mentioned in the methods, for the purpose of comparisons among the nutrition assessment tools, this study set a mandatory cutoff for each nutrition assessment tool. Thus, patients were distinctly categorized into the normal nutrition or malnourished groups by each tool. This study examined GPS score of $0 v s$. GPS score of 1 and 2, whereas previous studies have evaluated the three different scores independently. As a result, the significance of in-hospital mortality of GPS in this study was statistically underestimated. Thus, it may be implied that among the nutrition assessment tools, GPS was found to be prominently associated with short-term postoperative risks of all aspects in patients with stage III GC.

In locally advanced GC, albumin and CRP levels may be evaluated alternatively alongside nutrition and common 
prognostic guides. In addition to GPS, lower albumin levels also showed statistical significance with all the other nutrition assessment tools. Elevated CRP levels were also associated with malnutrition in the MUST, NRS, and PNI. While these nutrition assessment tools showed prominence in other fields of practice, correlation of albumin and CRP with these nutrition assessment tools suggested that they are accountable nutritional evaluation parameters. Thus, by combining the two parameters, GPS served as a reliable nutrition assessment tool with good prognostic value. Our study also revealed that elevated GPS is also significantly associated with some of the most common prognostic tools, such as body weight loss, ECOG, and tumor stage. This association may be attributed to the fact that these factors along with GPS are reflective of either tumor burdens, malnutrition, or disease severity, all of which lead to poor prognosis. Our previous study used multivariate analysis to show that GPS correlates with postoperative events independently of BWL, ECOG, and tumor stage (24). Thus, we suggest that GPS can be used as an additional guide for predicting prognosis of locally advanced GC.

While this study did not compare the five nutrition assessment tools' role in predicting overall survival, we believe that if a nutrition assessment tool does not provide postoperative prognostic benefit, its contribution to overall survival may be even more limited. As our previous study has explored the role of GPS in both short-term postoperative and long-term survival settings, the present study solidifies GPS's prognostic value among the different nutrition assessment tools.

Regardless of its strengths, this study has several limitations. First, as an analysis that relied partially on accurate record keeping, the retrospective design of this study had limited control over standardizing nutrition evaluation and initial outcome assessment. Second, given its single-center design, the outcome of this study may need to be externally validated, especially to confirm the performance of the PG-SGA in predicting postoperative adverse events in patients with GC as it showed no significant prognostic value in this study. Furthermore, patient selection bias may be present since patients with operable GC who decided against surgery and patients in whom metastasis was discovered perioperatively were excluded. With GPS's easy accessibility, future study goals may extend the use of GPS in patients with early GC or cancer of other origins.

\section{Conclusion}

Among the five commonly used nutrition assessment tools, GPS is most prominently associated with short-term postoperative risks of all parameters in patients with stage III GC. Furthermore, as it incorporates only objective values, the GPS is easily accessible during clinical practice. This study supports the application of GPS in locally advanced GC patients prior to surgery.

\section{Funding}

This research was not funded by any public, commercial, or nonprofit agency.

\section{Conflicts of Interest}

The Authors declare that no competing interests exist in relation to this study.

\section{Authors' Contributions}

Conception and design: Hsueh SW, Hsueh WH, Chou WC; Provision of study materials or patients: Liu KH, Tsai CY, Hsu JT; Collection and assembly of data: Hung CY, Tsang NM.

Data analysis and interpretation: Yang C, Chou WC; Final approval of manuscript: All Authors.

\section{Acknowledgements}

The Authors thank Ms. Vengi Ho and the members of the Cancer Center, Chang Gung Memorial Hospital for their assistance with data collection.

\section{References}

1 Eusebi LH, Zagari RM and Bazzoli F: Epidemiology of Helicobacter pylori infection. Helicobacter 19: 1-5, 2014. PMID: 25167938. DOI: $10.1111 /$ hel.12165

2 Bray F, Ferlay J, Soerjomataram I, Siegel RL, Torre LA and Jemal A: Global cancer statistics 2018: GLOBOCAN estimates of incidence and mortality worldwide for 36 cancers in 185 countries. CA Cancer J Clin 68(6): 394-424, 2018. PMID: 30207593. DOI: $10.3322 / \mathrm{caac} .21492$

3 Wadhwa R, Taketa T, Sudo K, Blum MA and Ajani JA: Modern oncological approaches to gastric adenocarcinoma. Gastroenterol Clin North Am 42(2): 359-369, 2013. PMID: 23639645. DOI: 10.1016/j.gtc.2013.01.011

4 Roukos DH and Kappas AM: Perspectives in the treatment of gastric cancer. Nat Clin Pract Oncol 2(2): 98-107, 2005. PMID: 16264882. DOI: 10.1038/ncponc0099

5 Cerantola Y, Grass F, Cristaudi A, Demartines N, Schäfer M and Hübner M: Perioperative nutrition in abdominal surgery: recommendations and reality. Gastroenterol Res Pract 2011: 739347, 2011. PMID: 21687620. DOI: 10.1155/2011/739347.

6 Muliawati Y, Haroen $\mathrm{H}$ and Rotty LW: Cancer anorexia cachexia syndrome. Acta Med Indones 44(2): 154-162, 2012. PMID: 22745148.

7 Santarpia L, Contaldo F and Pasanisi F: Nutritional screening and early treatment of malnutrition in cancer patients. J Cachexia Sarcopenia Muscle 2(1): 27-35, 2011. PMID: 21475618. DOI: 10.1007/s13539-011-0022-x

8 Takahashi T, Saikawa Yand Kitagawa Y: Gastric cancer: current status of diagnosis and treatment. Cancers (Basel) 5(1): 48-63, 2013. PMID: 24216698. DOI: 10.3390/cancers5010048 
9 Guo S and Dipietro LA: Factors affecting wound healing. J Dent Res 89(3): 219-229, 2010. PMID: 20139336. DOI: 10.1177/ 0022034509359125

10 Rosania R, Chiapponi C, Malfertheiner P and Venerito M: Nutrition in patients with gastric cancer: an update. Gastrointest Tumors 2(4): 178-187, 2016. PMID: 27403412. DOI: 10.1159/ 000445188

11 Inamoto S, Kawada K, Okamura R, Hida K and Sakai Y: Prognostic impact of the combination of neutrophil-tolymphocyte ratio and Glasgow prognostic score in colorectal cancer: a retrospective cohort study. Int J Colorectal Dis 34(7): 1303-1315, 2019. PMID: 31177316. DOI: 10.1007/s00384-01903316-z

12 Ikuta S, Sonoda T, Aihara T, Nakajima T and Yamanaka N: The preoperative modified Glasgow prognostic score for the prediction of survival after pancreatic cancer resection following non-surgical treatment of an initially unresectable disease. Contemp Oncol (Pozn) 22(4): 229-235, 2018. PMID: 30783386. DOI: $10.5114 /$ wo 2018.81344

13 Kimura S, D' Andrea D, Soria F, Foerster B, Abufaraj M, Vartolomei MD, Iwata T, Karakiewicz PI, Rink M, Gust KM, Egawa S and Shariat SF: Prognostic value of modified Glasgow Prognostic Score in non-muscle-invasive bladder cancer. Urol Oncol 37(3): 179.e19-179.e28, 2019. PMID: 30580906. DOI: 10.1016/j.urolonc.2018.11.005

14 Nakamura K, Nakayama K, Minamoto T, Ishibashi T, Sanuki K, Yamashita H, Ono R, Sasamori H, Komatsu-Fujii T, Ishikawa M and Kyo S: High preoperative Glasgow prognostic score is a negative prognostic factor for patients with endometrial carcinoma. Mol Clin Oncol 8(3): 429-433, 2018. PMID: 29456849. DOI: $10.3892 / \mathrm{mco} .2018 .1551$

15 Inamoto T, Matsuyama H, Sakano S, Ibuki N, Takahara K, Komura K, Takai T, Tsujino T, Yoshikawa Y, Minami K, Nagao $\mathrm{K}$, Inoue $\mathrm{R}$ and Azuma $\mathrm{H}$ : The systemic inflammation-based Glasgow Prognostic Score as a powerful prognostic factor in patients with upper tract urothelial carcinoma. Oncotarget $8(68)$ : 113248-113257, 2017. PMID: 29348903. DOI: 10.18632/ oncotarget.22641

16 Wakahara T, Ueno N, Maeda T, Kanemitsu K, Yoshikawa T, Tsuchida S and Toyokawa A: Is the Glasgow Prognostic Score applicable to both early- and advanced-stage gastric cancers. Gastroenterology Res 10(6): 359-365, 2017. PMID: 29317944. DOI: $10.14740 / \mathrm{gr} 943 \mathrm{w}$

17 Pacelli F, Bossola M, Rosa F, Tortorelli AP, Papa V and Doglietto GB: Is malnutrition still a risk factor of postoperative complications in gastric cancer surgery. Clin Nutr 27(3): 398407, 2008. PMID: 18436350. DOI: 10.1016/j.clnu.2008.03.002

18 Rey-Ferro M, Castaño R, Orozco O, Serna A and Moreno A: Nutritional and immunologic evaluation of patients with gastric cancer before and after surgery. Nutrition 13(10): 878-881, 1997. PMID: 9357024. DOI: 10.1016/s0899-9007(97)00269-4
19 Takeno S, Hashimoto T, Shibata R, Maki K, Shiwaku H, Yamana I, Yamashita R and Yamashita Y: Improvement of highsensitivity inflammation-based Glasgow prognostic score by gastrectomy is a favorable prognostic factor in patients with gastric cancer. Anticancer Res 34(10): 5695-5702, 2014. PMID: 25275076.

20 Chao PC, Chuang HJ, Tsao LY, Chen PY, Hsu CF, Lin HC, Chang CY and Lin CF: The Malnutrition Universal Screening Tool (MUST) and a nutrition education program for high risk cancer patients: strategies to improve dietary intake in cancer patients. Biomedicine (Taipei) 5(3): 17, 2015. PMID: 26264480. DOI: $10.7603 / \mathrm{s} 40681-015-0017-6$

21 Kondrup J, Rasmussen HH, Hamberg O and Stanga Z: Nutritional risk screening (NRS 2002): a new method based on an analysis of controlled clinical trials. Clin Nutr 22(3): 321-336, 2003. PMID: 12765673. DOI: 10.1016/s0261-5614(02)00214-5

22 Bauer J, Capra S and Ferguson M: Use of the scored PatientGenerated Subjective Global Assessment (PG-SGA) as a nutrition assessment tool in patients with cancer. Eur J Clin Nutr 56(8): 779-785, 2002. PMID: 12122555. DOI: 10.1038/sj.ejcn. 1601412

23 Lee JY, Kim HI, Kim YN Hong JH, Alshomimi S, An JY, Cheong JH, Hyung WJ, Noh SH and Kim CB: Clinical significance of the prognostic nutritional index for predicting short-and long-term surgical outcomes after gastrectomy: a retrospective analysis of 7781 gastric cancer patients. Medicine (Baltimore) 95(18): e3539, 2016. PMID: 27149460. DOI: 10.1097/MD.0000000000003539

24 Hsueh SW, Liu KH, Hung CY, Kuo YC, Tsai CY, Hsu JT, Hung YS, Tsang NM and Chou WC: Significance of the Glasgow Prognostic Score in predicting the postoperative outcome of patients with stage III gastric cancer. J Clin Med 8(9): E1448, 2019. PMID: 31547247. DOI: 10.3390/jcm8091448

25 Clavien PA, Barkun J, de Oliveira ML, Vauthey JN, Dindo D, Schulick RD, de Santibañes E, Pekolj J, Slankamenac K, Bassi C, Graf R, Vonlanthen R, Padbury R, Cameron JL and Makuuchi M: The Clavien-Dindo classification of surgical complications: five-year experience. Ann Surg 250(2): 187-196, 2009. PMID: 19638912. DOI: $10.1097 /$ SLA.0b013e3181b13ca2

26 Ryu SW and Kim IH: Comparison of different nutritional assessments in detecting malnutrition among gastric cancer patients. World J Gastroenterol 16(26): 3310-3317, 2010. PMID: 20614488. DOI: $10.3748 /$ wjg.v16.i26.3310

Received May 1, 2020

Revised May 15, 2020

Accepted May 16, 2020 